\title{
Long-term Outcome of Surgical Treatment of Intracavernous Giant Aneurysms
}

\author{
Isao DATE and Takashi OHMOTo
}

Department of Neurological Surgery, Okayama University Medical School, Okayama

\begin{abstract}
A number of approaches have been proposed for the treatment of intracavernous giant aneurysms. In the present study, we have analyzed long-term surgical outcome of 27 consecutive cases of our experience. All the cases were unruptured and symptomatic, showing symptoms such as extraocular movement disorder or visual disturbances. Thirteen cases were male and 14 cases were female. The age of the patients ranged between 11 and 75 years (average 52.2 years) and follow-up periods were between 1 and 20 years (average 7.7 years). Abducens nerve was disturbed in 20 cases, oculomotor nerve in 12 cases, optic nerve in six cases, trigeminal nerve in six cases, and trochlear nerve in five cases. In addition to conventional angiography, three-dimensional computed tomographic angiography, balloon test occlusion (BTO), slow injection angiography, aneurysmography, and single photon emission computed tomography with BTO were used to determine a method of treatment. Therapeutic modalities of the present series were as follows: four cases were unoperated, common carotid artery ligation was performed in eight cases, internal carotid artery (IC) ligation in three cases, IC ligation plus superficial temporal artery (STA)-middle cerebral artery (MCA) anastomosis in four cases, IC ligation plus high flow vein bypass in three cases, IC trapping plus STA-MCA anastomosis in three cases, and direct clipping in two cases. Although two cases showed early and late ischemic complications, other cases demonstrated improvement of cranial nerve dysfunction relatively soon after surgical treatment and long-term outcome was generally good. It is concluded that good long-term surgical outcome is obtained for intracavernous giant aneurysms by selecting adequate surgical treatment based upon careful preoperative evaluation of these aneurysms using sophisticated diagnostic methods.
\end{abstract}

Key words: cerebral aneurysm, intracavernous, giant, long-term, surgery

\section{Introduction}

Common carotid (CC) or internal carotid artery (IC) ligation has been the main surgical treatment for intracavernous giant aneurysms. ${ }^{8,22,29)}$ Recently, IC ligation combined with bypass surgery or direct clipping of the aneurysm has also been reported because of the development of preoperative cerebral blood flow reserve evaluation, the accumulation of the knowledge of the cavernous sinus microsurgical anatomy, or the development of the microsurgical techniques. ${ }^{1-6,9,10,12,24,28)}$ We have treated 27 cases of the intracavernous giant aneurysms by several surgical options and obtained good surgical outcome. The purpose of the present study is to analyze longterm surgical outcome of our experience.

\section{Materials and Methods}

We have analyzed 27 consecutive cases of the in- tracavernous giant aneurysms $(2.5 \mathrm{~cm}$ or larger $)$ treated so far. All the cases were unruptured and symptomatic, showing symptoms such as extraocular movement disorder or visual disturbances. Thirteen cases were male and 14 cases were female. The age of the patients ranged between 11 and 75 years (average 52.2 years) and follow-up periods were between 1 and 20 years (average 7.7 years). Regarding the disturbed cranial nerves, abducens nerve was involved in 20 cases, oculomotor nerve in 12 cases, optic nerve in six cases, trigeminal nerve in six cases, and trochlear nerve in five cases (Table 1).

In order to determine the therapeutic methods, preoperative evaluation was performed by a number of examinations as follows: digital subtraction angiography, three-dimensional computed tomographic (CT) angiography, slow injection angiography, aneurysmography, balloon test occlusion (BTO) (30 minutes) or Matas' test (manual), and single photon emission computed tomography (SPECT) with BTO. 
Table 1 Summary of 27 patients with intracavernous giant aneurysms

All symptomatic, unruptured

Size: $25-35 \mathrm{~mm}$ (average 30.3 )

Male: female $=13: 14$

Age: 11-75 yrs old (average 52.2)

Follow-up period: $1-20$ yrs (average 7.7 )

Disturbed cranial nerves preoperatively optic nerve oculomotor nerve trochlear nerve trigeminal nerve abducens nerve
6 cases

12 cases

5 cases

6 cases

20 cases

In addition to these examinations, the age of the patient as well as the existence of other systemic disease were considered to determine the therapeutic methods.

Therapeutic modalities of the present series were as follows: four cases were unoperated because of the patient's age or other diseases, CC ligation was performed in eight cases, IC ligation in three cases, IC ligation plus superficial temporal artery (STA)middle cerebral artery (MCA) anastomosis in four cases, IC ligation plus high flow vein bypass in three cases, IC trapping plus STA-MCA anastomosis in three cases, and direct clipping in two cases.

\section{Results}

There were four unoperated cases. The age of the patient and the existence of other systemic disease were the reasons for conservative treatment. Followup periods ranged between 5 and 11 years and the size of the aneurysm and the neurological symptoms

did not change during follow-up periods (Table 2).

There were eight cases treated by CC ligation. All the cases except one showed complete (4 cases) or partial ( 3 cases) recovery of neurological symptoms. One case showed complete recovery of abducens nerve palsy after surgery and spent normal daily life for 5 years when he suddenly suffered from cerebral infarction probably due to the migrated thrombus. Long-term outcome of other seven cases was good (Table 2).

Three cases were treated by IC ligation. All the three cases showed complete recovery of the neurological symptoms after surgery and the long-term outcome was good (Table 2).

Four cases were treated by IC ligation plus STAMCA anastomosis. Complete (3 cases) or partial (1 case) recovery of neurological symptoms was observed after surgery. The long-term outcome was good in all cases (Table 2).

There were three cases treated by IC ligation plus high flow vein bypass. These cases were young cases in their 20s and 30s and saphenous vein was used for external carotid (EC)- $\mathrm{M}_{2}$ high flow bypass. Two cases showed complete and one case showed partial recovery of neurological symptoms after surgery. The long-term outcome was good in all cases (Table 2).

There were three cases treated by IC trapping plus STA-MCA anastomosis. These cases were the cases in which direct clipping was initially tried but was not accomplished, then IC trapping (cervical IC and immediately distal to the aneurysm site) plus STAMCA anastomosis was performed. Two cases showed complete recovery of neurological symptoms after surgery. One case showed cerebral infarction in the MCA territory after surgery because

Table 2 Therapeutic modalities and surgical outcome

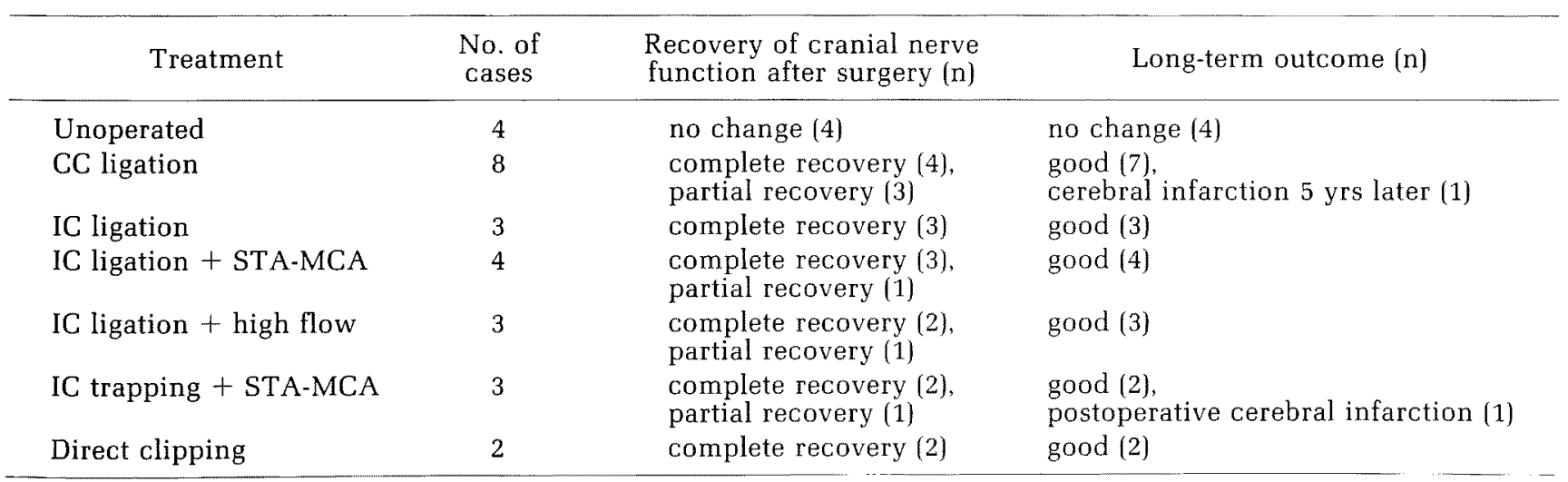

CC ligation: common carotid artery ligation, IC ligation: internal carotid artery ligation, STA-MCA: superficial temporal artery-middle cerebral artery anastomosis, high flow: high flow bypass using external carotid- $\mathrm{M}_{2}$ saphenous vein graft. 
the temporary occlusion time of the IC to try direct clipping of the aneurysm was too long (Table 2).

Two cases were treated by direct clipping of the aneurysm. In both cases the neck of the aneurysm was located on $\mathrm{C}_{3}$ portion (anterior siphon knee) of the IC. Trapping-evacuation technique ${ }^{30}$ was used to obtain decompression of the aneurysmal dome. Aneurysmal wall was opened to confirm the inflow and outflow orifices from the interior and direct clipping was performed. Preoperative abducens nerve palsy disappeared after surgery in both cases and the long-term outcome was good (Table 2).

\section{Illustrative Cases}

Case 1: A 50-year-old male experienced gradual deterioration of diplopia for a couple of months and was admitted to our ward. The neurological examination revealed that the patient had left abducens nerve palsy. CT scan and angiography demonstrated a left thrombosed intracavernous aneurysm $35 \mathrm{~mm}$ in size (Fig. 1A-E). BTO of the patient showed no neurological symptoms but SPECT with BTO demonstrated apparent reduction of cerebral blood flow in the left cerebral hemisphere. Left IC ligation plus STA-MCA anastomosis was performed (Fig. 1F, G). The patient's abducens nerve palsy disappeared 2 weeks after surgery and postoperative magnetic resonance (MR) imaging confirmed thrombosis of the aneurysm.

Case 2: A 34-year-old male complained of diplopia and was admitted to our ward. The neurological examination revealed that the patient had right abducens nerve palsy. Angiography demonstrated an intracavernous aneurysm $28 \mathrm{~mm}$ in size (Fig. 2A, B) and slow injection angiography revealed that the neck of the aneurysm was located on $\mathrm{C}_{3}$ and $\mathrm{C}_{4}$ portion of the IC (Fig. 2C-F). Although the patient did not show any neurological symptoms during BTO, the SPECT with BTO showed decreased cerebral blood flow in the right cerebral hemisphere (Fig. 2G, $\mathrm{H}$ ). Considering the age of the patient, right IC ligation plus high flow vein bypass was performed. The abducens nerve palsy disappeared 1 week after surgery and postoperative angiography disclosed patency of the EC-M $\mathrm{M}_{2}$ bypass (Fig. 2I). Postoperative MR imaging showed that the aneurysm was thrombosed (Fig. 2J, K).

Case 3: A 56-year-old female complained of diplopia for the past 3 months and neurological examination on admission disclosed her right abducens nerve palsy. Angiography revealed that the patient had a right intracavernous giant aneurysm $32 \mathrm{~mm}$ in size. Slow injection angiography revealed that the neck of the aneurysm was located on $\mathrm{C}_{3}$ portion of

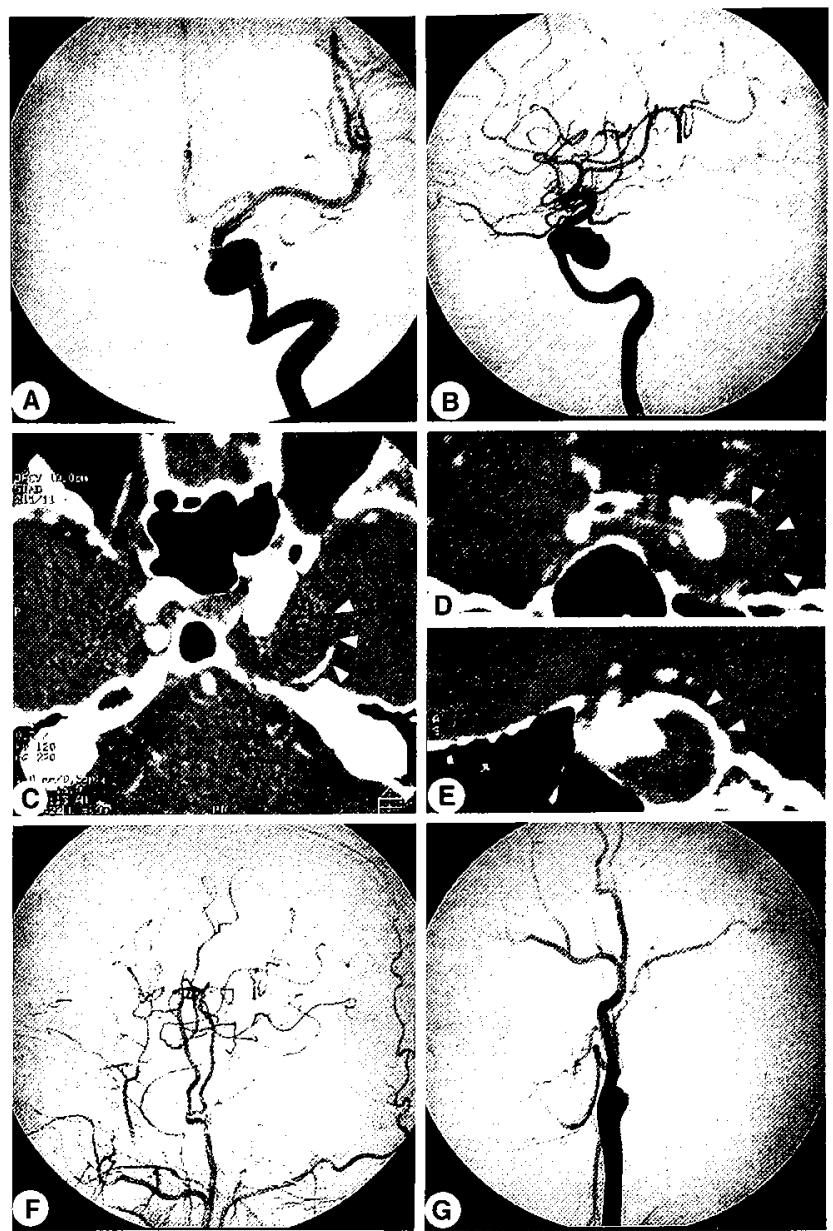

Fig. 1 Case 1, a 50-year-old male with left abducens nerve palsy. A, B: Digital subtraction angiograms (A: anteroposterior view, B: lateral view) show a left thrombosed intracavernous aneurysm $35 \mathrm{~mm}$ in size. CE: Postcontrast helical computed tomography scans (C: axial view, D: coronal view, E: sagittal view) show a giant thrombosed aneurysm. Only part of the aneurysm was enhanced by contrast material. Arrowheads show an outline of the aneurysm. F, G: Postoperative angiograms (F: lateral view, intracranial; $G$ : lateral view, cervical) demonstrate a patent superficial temporal artery-middle cerebral artery anastomosis (F) and complete ligation of the cervical internal carotid artery (G).

the IC (Fig. 3A-H) and direct clipping was performed (Fig. 3I, J). Her abducens nerve palsy disappeared after surgery and the long-term outcome was good. 


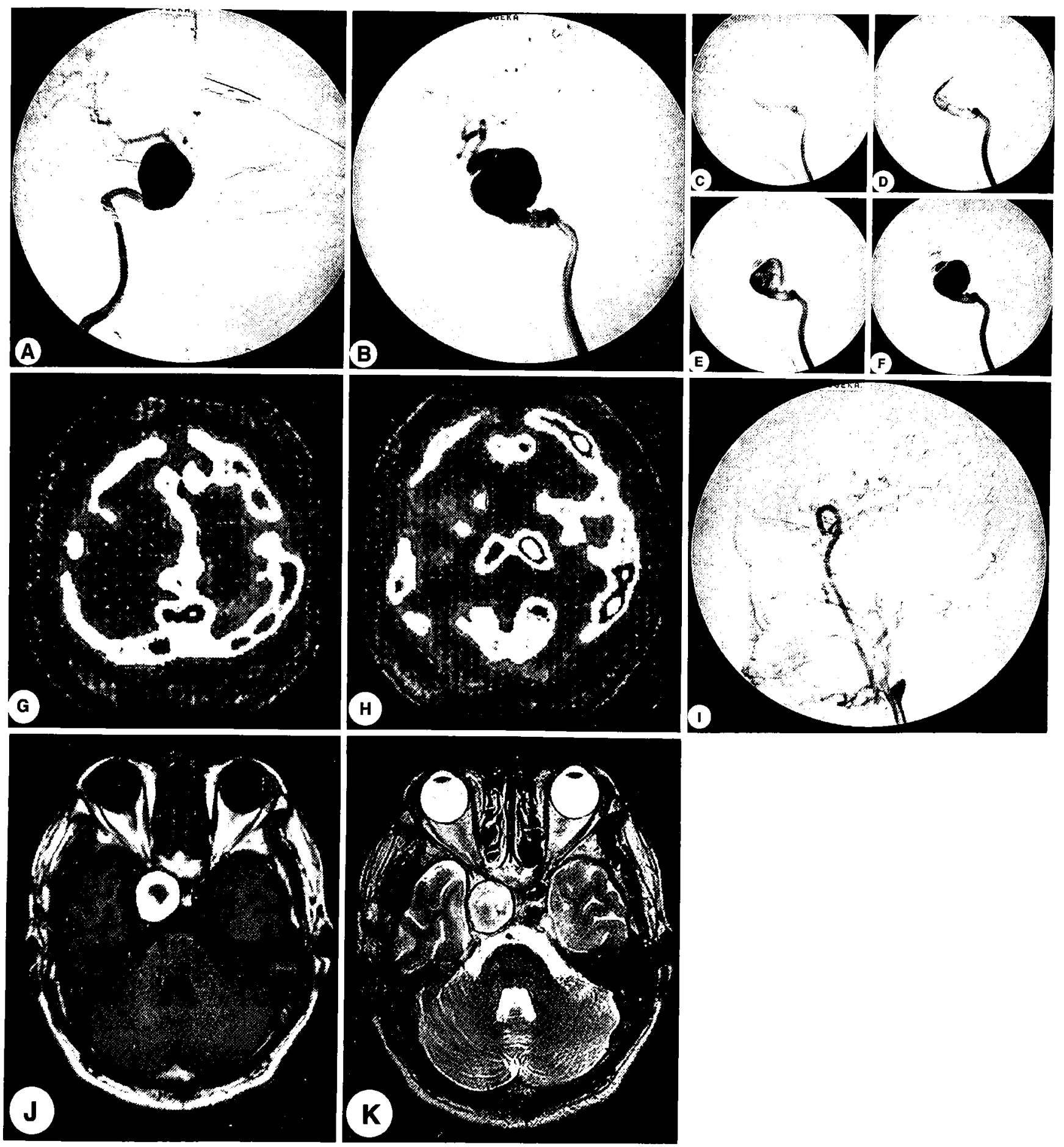

Fig. 2 Case 2, a 34-year-old male with right abducens nerve palsy. A, B: Digital subtraction angiograms demonstrate an intracavernous aneurysm $28 \mathrm{~mm}$ in size. C-F: Slow injection angiograms show the neck of the aneurysm is located on $C_{3}$ and $C_{4}$ portion of the right internal carotid artery (IC). G, H: Single photon emission computed tomography scans with balloon test occlusion show decrease of cerebral blood flow in the right cerebral hemisphere. I: Postoperative angiogram (lateral view) demonstrates the patent external carotid- $\mathrm{M}_{2}$ high flow bypass and complete occlusion of IC. J, K: Postoperative magnetic resonance images (J: $T_{1}$ weighted, $K: T_{2}$-weighted) show that the aneurysm is thrombosed. 


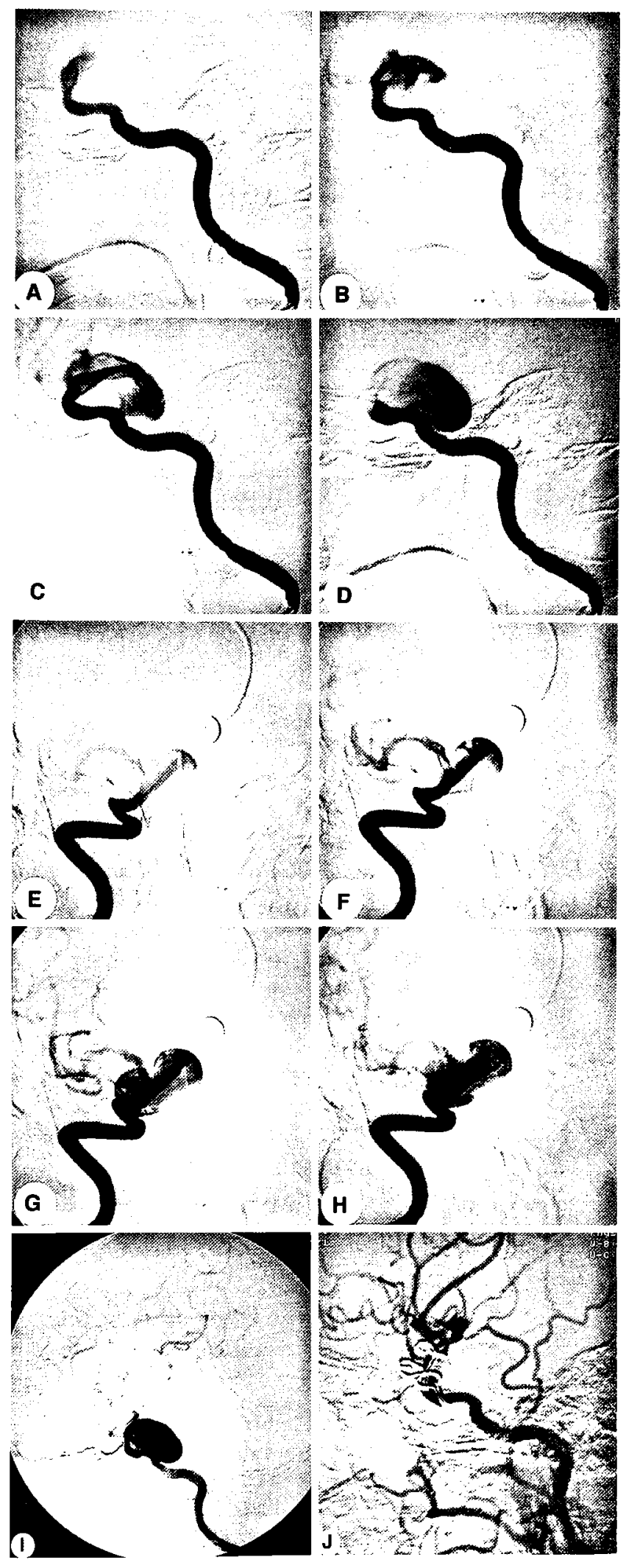

\section{Discussion}

Intracavernous aneurysms account for approximately $10 \%$ to $15 \%$ of all aneurysms originating from the IC. Female is more commonly affected and approximately $10 \%$ of cases show bilateral lesions. ${ }^{19)}$ Since natural history of the intracavernous aneurysm has been considered good, ${ }^{15)}$ there have been controversies over the surgical indication of this disease. Regarding the aneurysm location, the aneurysm part of which extends into the subarachnoid space or the aneurysm whose neck is located on $\mathrm{C}_{3}$ portions of the IC has been considered to have surgical indication. ${ }^{16]}$ Also, surgical treatment has been performed for the symptomatic intracavernous aneurysms showing progressive extraocular movement disorder, visual disturbance, and facial or orbital pain..$^{15,16)}$ Most of the studies of intracavernous aneurysms reported so far include asymptomatic or small aneurysms of this region ${ }^{18)}$ and there has been no study analyzing exclusively symptomatic and giant intracavernous aneurysms. In the present study, we show that good surgical outcome of intracavernous giant aneurysms could be obtained by careful preoperative evaluation and adequate surgical treatment.

There are a few studies analyzing natural history of the intracavernous aneurysms. ${ }^{13,15,17,18,20)}$ It should be noted that there are two reported cases which developed subarachnoid hemorrhage during follow-up periods. ${ }^{15,20)}$ Kupersmith et al. ${ }^{15)}$ reported that two cases among their 34 cases showed neurological deterioration during follow-up periods and Lye and $\mathrm{Jha}^{20)}$ reported such deterioration was observed in seven cases among their 20 cases. In our four cases of conservative treatment, there was no case with neurological deterioration or enlargement of the aneurysmal size. Although the prognosis of the aneurysms of this region is considered good from the viewpoint of mortality, we think surgical treatment should be considered for the cases with ex-

Fig. 3 Case 3, a 56-year-old female with right abducens nerve palsy. Conventional angiography demonstrates a right intracavernous giant aneurysm $32 \mathrm{~mm}$ in size. $\mathrm{A}-\mathrm{H}$ : Slow injection angiograms (A-D: lateral view, E-H: anteroposterior view) demonstrate that the neck of the aneurysm is located on $\mathrm{C}_{3}$ portion of internal carotid artery. I: Preoperative digital subtraction angiogram (DSA), lateral view. J: Postoperative DSA, lateral view, shows complete clipping of the aneurysm. 
traocular movement disorder or pain of the trigeminal nerve region.

A number of surgical approaches have been applied for the treatment of intracavernous aneurysms. CC ligation or IC ligation has been performed most commonly. ${ }^{8,22,29)}$ Regarding ischemic complications, Nishioka ${ }^{221}$ reported that $49 \%$ of cases with IC ligation and $28 \%$ of cases with CC ligation showed postoperative ische nic complications while Kak et al. ${ }^{14)}$ reported such complications occurred in $40 \%$ of IC ligation and $14 \%$ of CC ligation. Mount ${ }^{211}$ reported that CC ligation can be performed more safely than IC ligation but IC ligation promotes thrombosis of the aneurysm more rapidly than CC ligation. However, several reports mention that there are no such difference between CC ligation and IC ligation. ${ }^{11,23.26)}$ The postoperative complication such as thromboembolism from the clot in the IC is less frequent after $\mathrm{CC}$ ligation than after IC ligation probably because the collateral flow between IC and EC is considered to remain after CC ligation. ${ }^{29)}$ All of our cases of CC or IC ligation showed neurological improvement after surgery except one. However, it should be noted that one case was suffered from cerebral infarction 5 years after surgery, thus, the delayed ischemic complications cannot be neglected after CC or IC ligation. The delayed ischemic complications after $\mathrm{CC}$ or IC ligation have also been reported in the literature ${ }^{27.311}$ and chances of CC or IC ligation without bypass surgery have been decreasing in recent years.

Mainstay of surgical treatment for intracavernous giant aneurysms in recent years has been IC ligation (or IC occlusion by endovascular surgery) combined with STA-MCA anastomosis or EC-M $\mathrm{M}_{2}$ high flow bypass because microsurgical techniques developed and preoperative flow reserve can be analyzed more precisely by SPECT with BTO. ${ }^{2-6,10,12,24,28)}$ BTO or SPECT with BTO is performed preoperatively and low risk patients who have enough cerebral blood flow reserve commonly receive STA-MCA anastomosis and high risk patients who have less cerebral blood flow reserve commonly receive high flow bypass. ${ }^{2 \prime}$ In addition to this criteria, we usually perform high flow bypass for younger patients in their 20 s and 30s. Most of our cases of IC ligation with bypass surgery showed early postoperative improvement of neurological symptoms and long-term outcome was also good.

Accumulation of knowledge regarding microsurgical anatomy of the cavernous sinus region ${ }^{7,25)}$ supports the direct clipping of the aneurysms of this area. ${ }^{1,9)}$ As mentioned above, we performed direct clipping of the aneurysms of this region when the neck of the aneurysm was located on anterior si-

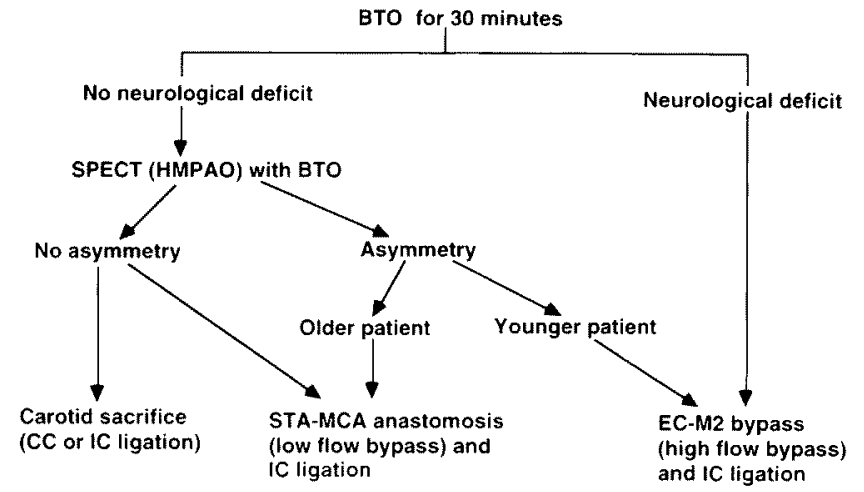

Fig. 4 Our treatment algorithm for the management of intracavernous giant aneurysms based upon balloon test occlusion (BTO). Direct clipping is applied in an aneurysm whose neck is located on the $C_{3}$ portion of the internal carotid artery (IC). CC: common carotid artery, EC: external carotid artery, HMPAO: hexamethyl-propyleneamine oxime, SPECT: single photon emission computed tomography, STA-MCA: superficial temporal artery-middle cerebral artery.

phon knee $\left(\mathrm{C}_{3}\right)$ portion of the IC. Trapping-evacuation technique ${ }^{300}$ is used to obtain decompression of the aneurysmal dome and aneurysmal wall is opened to confirm the inflow and outflow orifices from the interior. Two cases were treated by direct clipping with favorable results in the present study. We stress that slow injection angiography or aneurysmography is useful to judge the location of the neck and dome of the aneurysm as well as the size and the direction of the aneurysm.

Our current treatment protocol for management of intracavernous giant aneurysms is shown in Fig. 4. BTO is performed for 30 minutes and the cases showing neurological deficit during this period will receive IC ligation plus high flow bypass. SPECT with BTO is performed for the cases who do not show neurological deficit by BTO. Younger patients who show apparent asymmetry by SPECT with BTO will receive IC ligation plus high flow bypass and older patients who show apparent asymmetry by SPECT with BTO receive IC ligation plus STA-MCA anastomosis. The cases who do not show any asymmetry by SPECT with BTO will receive IC ligation plus STA-MCA anastomosis or CC or IC ligation only. Indication for direct clipping is determined in cases whose aneurysmal neck is located on the anterior siphon $\left(\mathrm{C}_{3}\right)$ of the IC by judging the result of BTO, slow injection angiography, and aneurysmography.

We conclude that good long-term surgical out- 
come can be obtained for intracavernous giant aneurysms by selecting adequate surgical treatment based upon careful preoperative evaluations of these aneurysms using sophisticated diagnostic methods.

\section{References}

1) Al-Rodhan NRF, Piepgras DG, Sundt TMJ: Transitional cavernous aneurysms of the internal carotid artery. Neurosurgery 33: 993-998, 1993

2) Barnett DW, Barrow DL, Joseph GJ: Combined extracranial-intracranial bypass and intraoperative balloon occlusion for the treatment of intracavernous and proximal carotid artery aneurysms. Neurosurgery 35: 92-97, 1994

3) Barrow DL, Cargill JCH: Aneurysms of the intracavernous carotid artery, in Tindall GT, Cooper PR, Barrow DL (eds): The Practice of Neurosurgery. Baltimore, Williams \& Wilkins, 1996, pp 2013-2032

4) Day AL, Masson RL, Knego RS: Surgical management of aneurysms and fistulas involving the cavernous sinus, in Schmidek HH, Sweet WH (eds): Operative Neurosurgical Techniques. Philadelphia, WB Saunders, 1995, pp 975-984

5) Diaz FG, Ausman JI, Pearce JE: Ischemic complications after combined internal carotid artery occlusion and extracranial-intracranial anastomosis. Neurosurgery 10: 563-570, 1982

6) Diaz FG, Ohaegbulam S, Dujovny M, Ausman JI: Surgical management of aneurysms in the cavernous sinus. Acta Neurochir (Wien) 91: 25-28, 1988

7) Dolenc V: Direct microsurgical repair of intracavernous vascular lesions. J Neurosurg 58: 824-831, 1983

8) Faria MJ, Fleischer AS, Spector RH: Bilateral giant intracavernous carotid aneurysms treated by bilateral carotid ligation. Surg Neurol 14: 207-210, 1980

9) Fukushima T, Day J, Tung H: Intracavernous carotid aneurysms, in Apuzzo MLJ (ed): Brain Surgery. New York, Churchill Livingstone, 1992, pp 925-944

10) Gelber BR, Sundt TJ: Treatment of intracavernous and giant carotid aneurysms by combined internal carotid ligation and extra- to intracranial bypass. $J$ Neurosurg 52: 1-10, 1980

11) Giannotta SL, McGillicuddy JE, Kindt GW: Gradual carotid artery occlusion in the treatment of inaccessible internal carotid artery aneurysms. Neurosurgery 5: 417-421, 1979

12) Heros RC, Nelson PB, Ojemann RG, Crowell RM, DeBrun G: Large and giant paraclinoid aneurysms: surgical techniques, complications, and results. Neurosurgery 12: 153-163, 1983

13) Inagawa $\mathrm{T}$ : Follow-up study of unruptured aneurysms arising from the $\mathrm{C} 3$ and $\mathrm{C} 4$ segments of the internal carotid artery. Surg Neurol 36: 99-105, 1991

14) Kak VK, Taylor AR, Gordon DS: Proximal carotid ligation for internal carotid aneurysms. A long-term follow-up study. J Neurosurg 39: 503-513, 1973

15) Kupersmith MJ, Hurst R, Berenstein A, Choi IS, Jafar
J, Ransohoff J: The benign course of cavernous carotid artery aneurysms. J Neurosurg 77: 690-693, 1992

16) Linskey ME, Sekhar LN, Hirsch WJ, Yonas H, Horton JA: Aneurysms of the intracavernous carotid artery: clinical presentation, radiographic features, and pathogenesis. Neurosurgery 26: 71-79, 1990

17) Linskey ME, Sekhar LN, Hirsch WJ, Yonas H, Horton JA: Aneurysms of the intracavernous carotid artery: natural history and indications for treatment. Neurosurgery 26: 933-937, 1990

18) Linskey ME, Sekhar LN, Horton JA, Hirsch WLJ, Yonas H: Aneurysms of the intracavernous carotid artery: a multidisciplinary approach to treatment. J Neurosurg 75: 525-534, 1991

19) Locksley HB: Natural history of subarachnoid hemorrhage, intracranial aneurysms and arteriovenous malformations. Based on 6368 cases in the cooperative study. J Neurosurg 25: 219-239, 1966

20) Lye RH, Jha AN: Unruptured aneurysms of the intracavernous internal carotid artery: outcome following carotid ligation or conservative treatment. $\mathrm{Br}$ J Neurosurg 3: 181-188, 1989

21) Mount LA: Results of treatment of intracranial aneurysms using the Selverstone clamp. J Neurosurg 25: 660-682, 1959

22) Nishioka H: Report on the cooperative study of intracranial aneurysms and subarachnoid hemorrhage. Section VII. I. Evaluation of the conservative management of ruptured intracranial aneurysms. I Neurosurg 25: 574-592, 1966

23) Odom GL, Tindall GT: Carotid ligation in the treatment of certain intracranial aneurysms. Clin Neurosurg 15: 101-116, 1968

24) Origitano TC, Al-Mefty O: Aneurysms of the cavernous sinus: treatment options and considerations, in Youmans JR (ed): Neurological Surgery. Philadelphia, WB Saunders, 1996, pp 1320-1334

25) Parkinson D: A surgical approach to the cavernous portion of the carotid artery: anatomical studies and case report. J Neurosurg 23: 474-483, 1965

26) Pozzati E, Fagioli L, Servadei F, Gaist G: Effect of common carotid ligation on giant aneurysms of the internal carotid artery: computerized tomography study. J Neurosurg 55: 527-531, 1981

27) Roski RA, Spetzler RF, Nulsen FE: Late complications of carotid ligation in the treatment of intracranial aneurysms. J Neurosurg 54: 583-587, 1981

28) Silvani V, Rainoldi F, Gaetani P, Bonezzi C, Rodriguez yBR: Combined STA/MCA arterial bypass and gradual internal carotid artery occlusion for treatment of intracavernous and giant carotid artery aneurysms. Acta Neurochir (Wien) 78: 142-147, 1985

29) Swearingen B, Heros RC: Common carotid occlusion for unclippable carotid aneurysms: an old but still effective operation. Neurosurgery 21: 288-295, 1987

30) Tamaki N, Kim S, Ehara K, Asada M, Fujita K, Taomoto K, Matsumoto S: Giant carotid-ophthalmic artery aneurysms: direct clipping utilizing the "trapping-evacuation" technique. I Neurosurg 74: 567- 
572,1991

31) Winn HR, Richardson AE, Jane JA: Late morbidity and mortality of common carotid ligation for posterior communicating aneurysms. A comparison to conservative treatment. J Neurosurg 47: 727-736, 1977
Address reprint requests to: I. Date, M.D., Department of Neurological Surgery, Okayama University Medical School, 2-5-1 Shikata-cho, Okayama 700-8558, Japan. 